yttria, found $Y t=90.12$, As their research is to be contintued, it would be unwise to use either of these investigations as a basis for changing the table. The lower of the two values appears to be the more probable.

Ruthenitm.-Vogt, ${ }^{1}$ from reductions of ruthenium dioxide finds $\mathrm{Ru}=$ 101.63.

Palladium.-Determinations of atomic weight by analysis of palladiammonium chloride have been made by Shinn. ${ }^{2}$ The mean value obtained was $\mathrm{Pd}=106.709$, but the individual determinations varied more than is satisfactory. Shinn supposes that the chloride is less definit than it has been assumed to be.

Radium.-From analyses of radium bromide, Hönigschmid ${ }^{3}$ finds $\mathrm{Ra}=225.97$, in confirmation of his former analyses of the chloride. The discordance between this value and the higher value obtained by others. is unexplained. The presumption is in favor of Hönigschmid's determination, but a change in the table may well be deferred until more evidence. is available.

The preceding table is that of 1913 , unchanged.

$$
\begin{aligned}
& \text { Signed, F. W. Clarke. } \\
& \text { T. E. Thorpe. } \\
& \text { W. Ostwald. } \\
& \text { G. Urrain. }
\end{aligned}
$$

\title{
THE ELECTRON CONCEPTION OF VALENCE. IV. THE CLASSI- FICATION OF CHEMICAL REACTIONS.
}

Bx J. M. NELSON, H. T. Beans aNd K. GEORGE Falk.

Received September 20, 1913.

Definition. ${ }^{4}$ The valence of an element may be defined as the number of corpuscles (negative electrons) an atom of that element loses or gains to form chemical bonds.

Assumptions Involved.-Every chemical bond formed between two atoms involves the transfer of a corpuscle from one atom to the other.

1 Sitzungsb. phys. med. Soz. Erlangen, 43, 268.

2 ThIs JOURNAL, 34, 1448.

3 Monats. Chem. 34, 283.

4 The electron conception of valence, as developed in the previous papers of this series, was applied in some detail to the structures of organic compounds and their reactions; while inorganic compounds were also included, although not discussed as thoroughly. In developing these valence views farther, and in attempting to extend them so as to include chemical reactions and relationships in general, it is necessary to state the fundamental definitions and conceptions in as clear a manner as possible. The definition of valence, and the views on oxidation and reduction which will be used as the fundathental conceptions, are, therefore, given in this paper and the classification of chemical reactions is then based upon them. It should hardly be necessary to state that the definition of valence and the consideration of oxidation and reduction as changes in the electrical state of certain definit atoms has received essentially the same treat- 
This transfer of a corpuscle requires that the atom which loses the corpuscle acquires a unit positive charge, the atom which gains the corpuscle a unit negative charge.

The existence of atoms and corpuscles is presupposed apparently. With regard to the former, essentially the same lines of reasoning may be followed if formula weights and combining weights are used in place of molecules and atoms. With regard to the assumption of the part played by corpuscles, the following may be pointed out. It has become customary to speak of positive and negative elements, and to base these terms upon contrasting or opposit influences on physical properties or behavior toward chemical reagents. The existence of ions in solution affords direct experimental evidence of positively and negatively charged particles, or in more specific terms, atoms and groups of atoms. For un-ionized substances and chiefly for organic substances, the use of the terms positive and negative as applied to atoms and groups of atoms, based upon more indirect evidence, has become firmly established. Furthermore, the same fundamental laws and relations may be expected to hold throughout chemistry, in spite of the customary convenient sub-divisions, due to the fact that different phenomena (such as ionization in solution) predominate more for one set of substances than for another. Positive and negative may then be taken to possess as real a significance for unionized substances as for ionized. Finally, even if the present theoretical views of valence could not have been developed without the conception of atoms and molecules, the whole subject of positive and negative relations as applied to the behavior of various classes of substances rests upon experimental bases which are independent of the assumption of the existence of corpuscles and atoms. The valence of an element may, therefore, also be defined as the number of equivalents of the acidic or of the basic constituents combined with, or associated with, one formula weight of that element. On the other hand, the introduction of the conception of corpuscles into the combinations of atoms permits of a convenient symbolism which alone would justify its use in practical work.

General Application.-The terms negative and positive elements convey a more or less definit meaning chemically, but with the valence con-

ment in publications by others, for the first time in a text-book, -as far as the writers are aware-by Ostwald in the first edition of his "Wissenschaftlichen Grundlagen der Analytischen Chemie," Leipzig, r9or. Stieglitz, in his "Qualitative Analysis," Part I, gives a clear account of these relations, which have also been taken up by Abegg, J. J. Thomson, W. A. Noyes, Boettger, and others. Unfortunately, however, even in those text-books in which the definitions and fundamental conceptions are stated in the most precise manner, the application to chemical changes has not been followed consistently in the further treatments. It is hoped that this paper may aid in pointing out the importance, not only of stating the fundamental conceptions, but also of applying them logically and consistently wherever possible. 
ception involving corpuscles the meaning becomes more readily understood and applied to specific instances. A negative element, or an element showing negative characteristics, would then be one in which an atom of that element, in virtue of being combined with another atom, receives one or more corpuscles (negative electrons) from that atom, one or more chemical bonds being produced in this way. Thus, in hydrochloric acid, chlorine is the negative element, in carbon monoxide, oxygen is the negative element, etc. Similarly a positive element. is one in which an atom loses one or more corpuscles to another atom, to form one or more chemical bonds.

For ionizable substances, the definition of valence for an atom or group of atoms is taken to be the number of faradays of electricity carried by the formula weight, in grams of that atom or group of atoms, which enables it to exist in the free state in solution as an ion. The definition of valence given at the beginning of this paper includes this class and extends it to include all substances, whether or not the atoms, or groups of atoms, present in them have been found to exist as ions in a solution.

The modern view of oxidation considers it to consist in the loss of negative charges of the atom oxidized or the gain of positive charges; and reduction, the loss of positive or gain of negative charges. Since the term "oxidation," as used in the past, is not limited to reactions involving oxygen, it is proposed to use the term aDDuctron ${ }^{1}$ for reactions in which the increase in the number of positive charges or the decrease in the number of negative charges of an atom or group of atoms is the main feature, including thereby oxidation, chlorination, etc. The term REDUCTION involving the decrease in the number of positive charges or the increase in the number of negative charges does not. necessarily involve the element hydrogen and can be retained in its present significance without confusion.

Strictly speaking, if the negative electron is considered to be the main factor in the formation of a chemical bond, a contradiction is involved in the terms proposed. In view, however, of the fact that the nomenclature can be based just as well upon the increase and decrease in the number of positive charges, and since it is desirable to cause as little confusion as possible of terms now in use, it is thought desirable not to introduce entirely new words to classify the phenomena. This is readily done by keeping the term reduction for reactions for which it has always been used, and by introducing the term adduction for reactions which involve the addition of positive charges to the atom, or group of atoms, in question, or for the reactions which heretofore have been included under oxidation.

Since corpuscles may be either given off or taken up by an atom, it is

${ }^{1}$ Suggested to the writers by Dr. M. L. Hamlin. 
evident that if the valence is positive in the former case, it is negative in the latter. If an atom gives up a corpuscle it shows a positive valence of one, if it gains a corpuscle it shows a negative valence of one. Thus in hydrochloric acid, the valence of the hydrogen is $+x$, of the chlorine $-I$; in ammonia, the valence of the nitrogen -3 , of the hydrogen.$+ I$; in carbon dioxide of the carbon +4 , of the oxygen -2 ; in methane, of the carbon -4 , of the hydrogen $+\mathrm{r}$; in phosphine, of the phosphorus -3 , of the hydrogen $+\mathrm{r}$; in phosphorus trichloride, of the phosphorus +3 , of the chlorine $-\mathrm{I}$; etc. The change from carbon monoxide to carbon dioxide involves the adduction of the carbon, or the addition of two positive charges to the carbon atom; the change from methane to carbon dioxide also the adduction of the carbon, or the addition of eight positive charges to the carbon atom; the reverse change from carbon dioxide (or carbon tetrachloride) to methane, the reduction of the carbon, or the removal of eight positive charges (or the addition of eight negative charges) to the carbon atom; etc. Similar relations may be pointed out for the compounds of nitrogen as well as for compounds of other elements. If, in place of considering positive and negative atoms and groups of atoms, the basic and acidic constituents of a substance are considered, the same relations appear.

General Classification of Reactions.-In the previous papers of this series ${ }^{1}$ in which the Electron Conception of Valence was applied to a number of different classes of compounds, many of them derived from the field of organic chemistry, the problem was treated from the statical side. The formulas assigned to different compounds, or to isomeric compounds of a definit composition, were derived as far as possible, it is true, from a study of the reactions of the substances in question, but the main problem attacked dealt with the most probable directions of the valences in the compounds and the general principles underlying these configurations.

In this paper an attempt will be made to classify chemical reactions on the basis of a few simple conceptions based upon the formation of chemical bonds by the transfer of electrons and the (electrical) state of the atoms involved. The general views of structure outlined in the previous papers will be used.

Since the production of every chemical bond involves the loss of (at least) one corpuscle by one atom and the gain of (at least) one corpuscle by another atom, this formation of a bond will involve a simultaneous adduction of one atom and reduction of the other. Thus, starting with a neutral hydrogen atom and a neutral chlorine atom, in order to form a molecule of hydrochloric acid the hydrogen atom loses a corpuscle or

${ }^{1}$ School of Mines Quarterly, 30, 79 (1909). ThIs Journal, 32, I637 (I910); 33, II40 (I9II). Orig. Communications VIII Intern. Congr. Applied Chemistry, 6, 212 (I912). J. pr. Chem., N. F., 88, 97 (I913). 
adds a positive charge, becoming adduced, and the chlorine atom gains a corpuscle or loses a positive charge becoming reduced; for example $\mathrm{H}^{+} \longrightarrow \mathrm{Cl}^{-}$, the arrow indicating the direction in which the corpuscle is transferred to form the bond as explained in the previous papers. This view can evidently be extended to the formation of all chemical bonds involving simultaneous reduction and adduction of the atoms united by the transfer of corpuscles.

In the classification to be given, the formation and decomposition of "onium" compounds plays an important part. A typical example of an onium compound is ammonium chloride, $\mathrm{NH}_{4} \mathrm{Cl}$, formed from ammonia, $\overline{\bar{N}} \hat{H}_{3}$, by the simultaneous addition to the nitrogen of a positive and a negative charge, or the loss by the nitrogen of a corpuscle to the chlorine from the hydrochloric acid, and the gain of a corpuscle by the nitrogen from the hydrogen from the hydrochloric acid. Onium compounds, in general, are made up similarly by the addition of an electropositive atom and an electronegative atom to an atom which is itself in combination with one or more electropositive atoms, or groups of atoms. In terms of corpuscles, an atom when combined with one or more atoms in virtue of having received corpuscles from them, may form an onium compound by the simultaneous loss and gain of a corpuscle in connection with other atoms, or, in other words, by acquiring a positive and a negative charge--the algebraic sum of these added charges being zero. Many of these onium compounds show typical reactions of salts, but are ordinarily dealt with in organic chemistry, since the positive atoms or groups present are generally hydrocarbon radicals. Among the representatives of these substances may be mentioned $\underset{=\mp}{\operatorname{ammonium}} \underset{=\mp}{\mathrm{NR}^{+}}{ }_{4} \mathrm{X}^{-}$, iodonium, $\mathrm{IR}_{2}{ }^{+} \mathrm{X}^{-}$, sulfonium, $\mathrm{SR}_{3}{ }^{+} \mathrm{X}^{-}$, arsonium, $\mathrm{AsR}_{4}{ }^{+} \mathrm{X}^{-}$, etc., compounds.

The proposed classification in its most general form may be stated as follows:

In every reaction, either

I. The algebraic sum of the positive and negative charges on a definit atom of the molecules involved changes; or

II. The algebraic sum of the positive and negative charges on a definit atom of the molecules involved remains constant.

If $I$, the algebraic sum changes; either $A$-the number of corpuscles on the atom in question increases; $i$. e., the number of negative charges on the atom is increased, or the number of positive charges on the atom is decreased (reduction); or B-the number of corpuscles on the atom in question decreases; $i . e$., the number of negative charges on the atom 
is decreased, or the number of positive charges on the atom is increased (adduction).

If II, the algebraic sum remains constant; either $A$-the arithmetical sum of the positive and negative charges on the atom in question changes; $i . e$, the atom in question gains or loses the same number of positive and negative charges simultaneously (onium compounds); or B-the arithmetical sum of the positive and negative charges on the atom in question remains constant; $i . e$. , the electric charge on the atom in question remains unchanged.

It would be manifestly impracticable here to apply the classification to the great number of reactions which are known in chemistry. The remainder of this article will therefore be devoted to a short discussion of the principles involved in connection with a few of the more common reactions.

It will be noted frst that the consideration of the changes involved in a chemical reaction is based upon the number of electric charges carried by an atom, and the changes in these numbers which accompany the reaction. Thus in adduction and reduction, certain definit atoms change their electric charges. In the formation of ferric chloride from ferrous chloride, the iron loses an additional electron, thus reaching a higher state of adduction (chlorination), the electron removed being taken up by an atom of chlorine. In this sense, it is incorrect to speak of the adduction of ferrous chloride to form ferric chloride, since the chlorine of the ferrous chloride remains unchanged and the additional chlorine added becomes reduced from the atomic (uncharged) state, only the iron becoming adduced. Similarly, in the reaction between stannous chloride and mercuric chloride, in which stannic chloride and mercurous chloride are formed, the change consists of the adduction (oxidation, gain of positive charges) of the stannous tin to form stannic tin at the same time that the mercuric mercury is reduced (loss of positive charges) to mercurous mercury; the character of the chlorine not changing throughout, although initially it may have been combined with the mercury and finally with the tin.

The general classification of chemical changes which depends upon the loss and gain of corpuscles by atoms may be summarized concisely and the reactions to which they apply stated in a simple manner. In every reaction; -I, If the algebraic sum of the positive and negative charges on a definit atom of the molecules changes; either, $A$, the number of corpuscles on the atom increases; or $B$, the number of corpuscles on the atom decreases; II, If the algebraic sum of the positive and negatice charges on the atom remains constant; either $A$, the arithmetical sum changes; or $B$, the arithmetical sum remains constant. Applied to chemical reactions, IA inclutes reduction reactions, IB adduction reactions, IIA onium compound formation, 
IIB metatheses in which none of the changes IA, IB, or IIA takes place. Stated slightly differently, the classification includes reactions involving reduction, oxidation, onium compound formation, and simple replacement or rearrangement.

Before considering special reactions, it will be necessary to take up briefly some general reactions by means of which the structures of various compounds may be ascertained. The general principles by which the structure of a given compound may be determined were given in the previous papers, and will not be repeated here. The main reaction to which it is desired to draw attention is that involving hydrolysis. ${ }^{1}$ In this reaction, when an atom or group is replaced by a hydrogen atom or hydroxyl group derived from water, unless there is very distinct evidence to the contrary, it may be assumed that no change in the electrical states of the atoms taking part in the reaction occurs. If acid or base is present and accelerates the hydrolysis, there may be a greater possibility of electrical change, but still unless there is evidence to the contrary, no change may also be assumed here. The reactions involving hydrolysis may therefore be used to throw light on the structures of substances, by converting them into related substances containing hydrogen and hydroxyl in place of the atoms or groups in question. These reactions belong to Group IIB. A similar group of reactions includes those in which the elements of water, as hydrogen and hydroxyl, are added to a definit atom to form onium compounds, or where the elements of water are removed from such onium compounds. These reactions belong to Group IIA. The algebraic sum of the corpuscles of the central atom in these reactions remains unchanged.

In taking up specific reactions, it will be convenient to take up Groups IA and IB, reduction and adduction, together, since these reactions are reversible. The reactions of these groups involve in every case a change in the electrical charge, or in the number of corpuscles of one or more atoms of the reacting molecules. Since the reduction or adduction of some one atom in a molecule is generally the main feature of such reaction, it is only the change in the state of this atom which need be considered in the reaction, although, as stated before, reduction and adduction must always take place simultaneously, no electrical charges or corpuscles being created in any reaction. It is only by considering part of a reaction that changes in electrical charges of atoms can be spoken of. The adduction of certain atoms in the molecules is shown in the following changes:

${ }^{1}$ Hydrolytic reactions were used for determining the electric charges on certain atoms by Selivanow, Ber., 25, 3617 (1892); Stieglitz, Am. Chem. J., 18, 756 (1896); W. A. Noyes, This Journal, 35, 769 (1913); and others. 


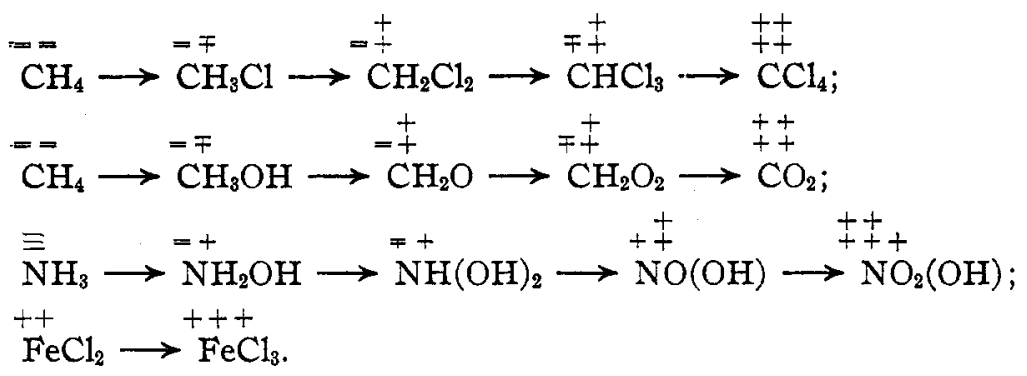

In each of these transformations, the change in the electrical charge of only one atom is produced, the carbon atom, the nitrogen atom, and the iron atom being adduced. At the same time a reduction of some other atom which is not shown in the scheme has taken place. The reversal of these reactions would evidently show reduction of the atoms considered.

The reactions classed under Group IIA include the formation of onium compounds. Under this name are classed the reactions in which both a positive and a negative atom or group of atoms are added to an atom so that while the arithmetical sum of the positive and negative charges on the atom changed, the algebraic sum remains unchanged. Thus $\mathrm{H}^{+} \mathrm{Cl}^{-}$added to $\mathrm{N} \equiv \mathrm{H}_{3}{ }^{+}$forms the ammonium salt $\mathrm{NH}^{+} \mathrm{Cl}^{-}$. The formulas for the carbonium compounds, ${ }^{=\mp} \mathrm{CR}_{3}+\mathrm{X}^{-}$, show them to be derived from methylene compounds $\mathrm{C}^{=} \mathrm{R}_{2}^{+}$similarly by the addition of $\mathrm{R}^{+} \mathrm{X}^{-}$. The ionizable form of triphenylmethyl chloride may serve as an example. The existence of these carbonium compounds suggests the possibility of onium compounds derived from methane derivatives and possessing the general type formula $\mathrm{CR}_{5}{ }^{+} \mathrm{X}^{-}$. These might appropriately be termed carbononium compounds. There is no direct evidence at hand that these compounds exist, but in the third paper of this series, the possibility of the transient existence of compounds containing such hexavalent carbon atoms was suggested in discussing the reciprocal transformations of tautomeric substances. A study of the hydrogen, oxygen, and onium compounds of the fourth, fifth, sixth, and seventh groups of the Periodic System also indicates the probable existence of such hexavalent carbon atoms. The assumption of the existence of hexavalent onium carbon may evidently be used to explain the course of a number of organic reactions, as for instance, substitutions, but a further consideration of these views will be deferred to a later paper.

It may be mentioned that Group IIA also includes the reverse reactions in which both a positive and a negative atom or group are removed from onium compounds.

The group of reactions IIB includes those reactions in which the elec- 
trical charges on the atoms taking part in the reaction remain unchanged. Among the reactions included in this group may be mentioned the mixing of salt solutions, precipitations, replacements, and in fact all ionic reactions which do not involve reduction or adduction. The simple hydrolyses, spoken of before, are included in this group as well as simple replacements such as that of one halogen by another in organic or inorganic compounds. The addition of hydrogen halides (not of halogens) to unsaturated organic compounds containing double or triple bonds, belong as a rule to this group. Rearrangements, such as tautomeric changes, are also included.

It would serve no useful purpose to attempt to apply the principles outlined to all classes of reactions in an article such as this. The remainder of this paper will be devoted to the consideration of the reactions of a few substances which appear to be of special interest for one reason or another.

The first substance which will be considered is hydrogen peroxide. A careful study of the reactions of this substance reveals the fact that it appears to act as a reducing agent only toward permanganate and manganese dioxide (in the presence of nitric acid). With silver oxide, hydrogen peroxide reacts to form silver peroxide first, and the silver peroxide then decomposes into silver and oxygen, so that although hydrogen peroxide and silver oxide react to form metallic silver apparently, the first action is a double decomposition. Other apparently reducing reactions of hydrogen peroxide are in the same way first metatheses or adductions, followed by the decomposition of the primary products of the reaction. The only exceptions appear to be the manganese reactions as stated, and it is conceivable that further study might show these to be only apparent exceptions. The question as to the structure of hydrogen peroxide and the reason for its adducing action is of interest. Hydrogen peroxide may be assigned either of the tautomeric formulas ${ }^{1}$



or

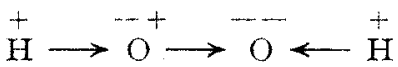

Since hydrogen is evolved as such, in none of the reactions, it retains its positive charge and is unchanged in the reaction. The whole change centers in the change in the electrical charge of the oxygen. Since it causes adduction in other substances, it must itself be reduced or lose positive charges. In formula (I) the change would be represented by the transformation of $\mathrm{O}$ into $\vec{O}$; in formula (2) by $\mathrm{O}^{+}$into $\overline{\mathrm{O}}$; or, in other words, the change in the valence of the one oxygen atom from zero to -2

Cf. III paper of this series. 
may be looked upon as the reason for the adducing action of hydrogen peroxide. ${ }^{1}$

The reactions of hydrogen peroxide serve to show how the valence views may be applied to an inorganic substance. The application to an organic reaction will be of interest and for this purpose the diazo reaction has been chosen. The general reaction as commonly given may be formulated as follows:

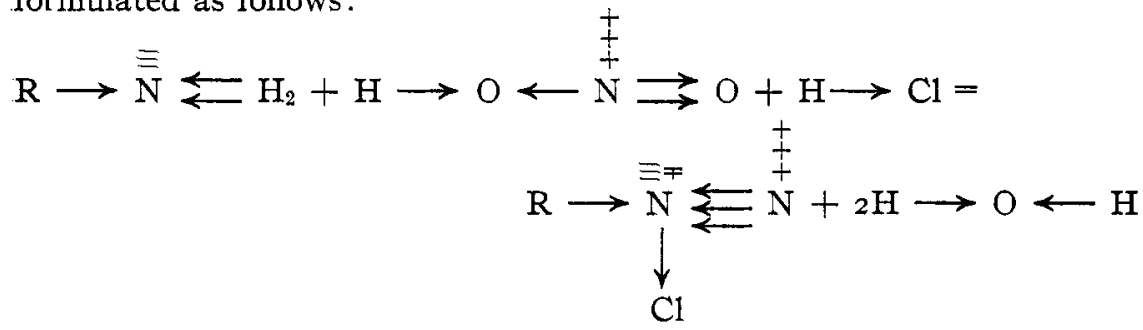

Approaching this reaction from the point of view of the classification of chemical reactions as given in this paper, it is evident that the first reaction when an amine and an acid are brought together in solution will be

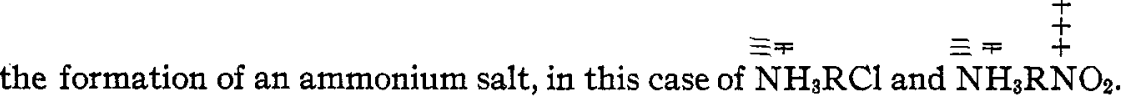
Since the latter salt is of main interest in this connection, it will be formulated in detail:

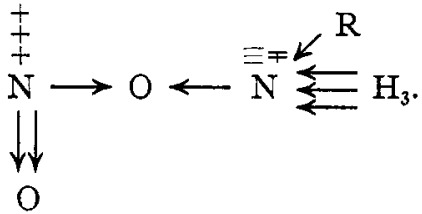

The structure of this ammonium salt is of the same type as the structure of ammonium acetate,

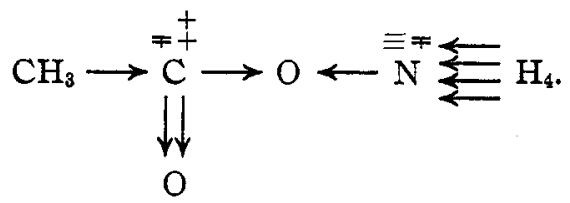

The latter substance loses the elements of water to form acetamide

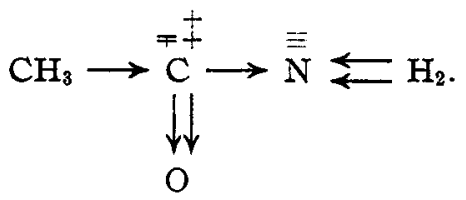

${ }_{1}$ The writers wish to take this opportunity to thank Professor Stieglitz for pointing out to them some of the electronic relations involved in the reactions in which hydrogen peroxide takes part. 
Similarly the former substance loses the elements of water to form a nitros: amine

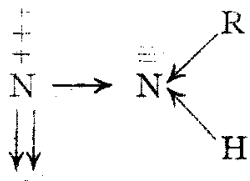

O

and then in the presence of the hydrochloric acid, simultaneous replacement and rearrangement into the diazonium salt,

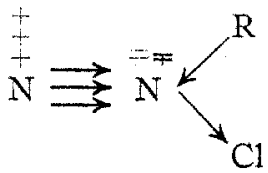

takes place. The formation of a diazo compound from an amine and nitrous acid has therefore been resolved into the following simple reactions: first, the formation of an onium compound belonging to reactions of Group IIA, secondly, the elimination of the elements of water from this onium compound (comparable to the formation of acetamide from ammonium acetate), belonging to the same group of reactions, and lastly the tautomeric change and simultaneous replacement of hydroxyl by chlorine and rearrangement of the nitrosamine into the diazonium salt, belonging to reactions of Groups IIA and IIB.

The expression "valence of a group of atoms" has been used a number of times in this paper. Although its meaning is evident, it is, strictly speaking, not a correct expression. The valence of such a group of atoms is always the valence of a particular atom present in the group. The other atoms combined with this atom only influence its valence, and while the particular atom which gives the valence number its value for that group, especially if the group is present in a whole series of compounds, is not always definitly known, in the final analysis the valence of the group as determined is always the valence of that atom.

This raises the question of the complex inorganic salts. If the views of Ramsay, which have been repeatedly quoted in connection with the structures of these substances, are followed, it is apparent that if, for example, the cobaltammine salts are chosen, their formation belongs to the reactions of Group IIA, $i . \varepsilon_{\text {., }}$ onium compound formation. The only difference as compared with the ordinary onium salt formation, is that two atoms in one molecule are involved, each losing a corpuscle to the other at the same time that one is received from the other. The formula of cobalt: hexammino trinitrite,

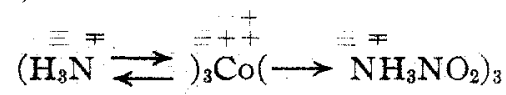

may serve as example. 
In conclusion, the writers wish to thank Dr. M. L. Hamlin for his aid in developing the classification of reactions presented here.

Columbia University,

HaRriman Research Laboratory, Roosevelt Hospital.

[Contributions from the Chemical laboratories of Columbia University, No. 220.]

\section{THE WEIGHT OF A FALLING DROP AND THE LAWS OF TATE, XV. THE DROP WEIGHTS OF CERTAIN ORGANIC LIQUIDS AND THE SURFACE TENSIONS AND CAPILLARY CONSTANTS CALCULATED FROM THEM. ${ }^{1}$}

By J. Livingston R. Morgan and Philip M. Chazal.

Received July 28, 1913.

Six tips were used in the course of this investigation, all of which were standardized against benzene. The mean values of the drop weights of benzene, together with the values calculated for $\mathrm{K}_{\mathrm{B}}$, by aid of the equation

$$
\mathrm{K}_{\mathrm{B}}=\frac{w(\mathrm{M} / d)^{2 / \mathrm{s}}}{\left(288.5-t_{\mathrm{o}}-6\right)^{2}}
$$

are given in Table $I$. In the last two columns of this table will also be found the constants, $k_{1}$, by which the drop weight from the tip must be multiplied in order to give the surface tension, $\gamma$, of the liquid in dynes per centimer at the temperature in question; and the constants $\left(k_{2}\right)$ by which the drop volume (drop weight/density) must be multiplied in order to give the capillary constant ( $a^{2}=$ height of ascension in a capillary tube $\times$ the radius of the bore of the tube) of the liquid, also, of course, at the temperature at which the drop weight is found.

TABle I.-STANDardization OF The TrPs.

$\begin{array}{cccccccc}\text { No. } & t_{0} . & d . & w(\mathrm{mg}) . & . w(\mathrm{M} / d)^{2 / 8} & \mathrm{~K}_{\mathrm{B} .} & k_{1 .} & k_{2 .} \\ \mathbf{I} & 30.0 & 0.86824 & 28.820 & 578.092 & \mathbf{2 . 2 8 9 5} & 0.9237 & 0.1886 \\ 2 & 30 . \mathrm{I} & 0.86813 & 28.760 & 577.100 & \mathbf{2 . 2 8 6 4} & 0.9249 & 0.1888 \\ 3 & 30.0 & 0.86824 & 28.415 & 569.700 & \mathbf{2 . 2 5 7 3} & 0.9367 & 0.1913 \\ 4 & 30.0 & 0.86824 & 28.985 & 581.400 & 2.3067 & 0.9168 & 0.1872 \\ 5 & 30.0 & 0.86824 & 29.224 & 586.240 & \mathbf{2 . 3 2 1 7} & \mathbf{0 . 9 1 0 9} & 0.1860 \\ 6 & 30.0 & 0.86824 & 29.352 & 588.800 & 2.3319 & 0.9069 & 0.1851\end{array}$

The mean diameters of these tips can be calculated, according to Morgan and Cann, ${ }^{3}$ from their $\mathbf{K}_{\mathbf{B}}$ values. Such a calculation leads to the following results: $D_{1}=5.420 ; D_{2}=5.413 ; D_{3}=5.344 ; D_{4}=5.461$; $\mathrm{D}_{5}=5.497 ; \mathrm{D}_{6}=5.52 \mathrm{I}$ millimeters.

${ }_{1}^{1}$ For other papers in this series, see This Journal, 30, 360, I055; 33, 349, 643, $657,672,1042,1060,1275,1713 ; 35,1249,1505,1750,1759$. In this paper, as in the other recent ones, only the means of the 30 -drop and 5 -drop determinations are given, in order to save space.

2 For details of the calibration in general see Turs Journal, 33, $7713-27$.

${ }^{3}$ ThIS JOURNAL, 33, 349-62. 